九州大学学術情報リポジトリ

Kyushu University Institutional Repository

\title{
Frequency Distribution and Average Chemical Composition of the Volcanic Rocks in Japan
}

Taneda, Sadakatu

Faculty of Science, Kyushu University

https://doi. org/10.5109/1526214

出版情報：九州大學理學部紀要：Series D, Geology. 12 (3)，pp.237-255，1962-06-11. Faculty of Science, Kyushu University バージョン:

権利関係 : 


\title{
Frequency Distribution and Average Chemical Composition of the Volcanic Rocks in Japan
}

By

\author{
Sadakatu TANEDA
}

\section{Contents}

\begin{abstract}
Introduction ...................... 237
I. Lavas of Japanese volcanoes as a whole . . . . . . . . . . 238

II. Grouping of lavas of Japanese volcanoes. . . . . . . . . . . 239

III. Lavas of each group . . . . . . . . . . . . . . . . . . 245

IV. Comparison of lavas of historic times and those of prehistoric times...................... 247

V. Comparison of active volcanoes and non-active volcanoes. . . . . 252

VI. Suggestion concerning the forecast of volcanism . . . . . . . 254 Acknowledgements . . . . . . . . . . . . . . 255

References .................... 255
\end{abstract}

\section{Introduction}

This is an attempt to estimate the fluctuation of lava activity in the last period of geological times, standing on geological petrological and volcanological standpoint.

I calculated the average chemical composition of effusive rocks of Japan (Taneda, 1952), using 334 analytical results, published through 1951. However during 10 yearss since 1951, many analytical results were published, making desirable the recalculation of the average. In this paper I considered 619 good analytical data of about 90 volcanoes in Japan. The number of analyses was more than 800 , but two or more data of the same lava flow or the same bomb-pumice bed were taken as average. All of the data used, except about twenty unpublished data of the Haruna and Aso volcanoes, have been published before May, 1961.

The data were divided into 4 groups (the lavas of historic times (1 group), the lavas of prehistoric times (2 groups) of active volcanoes and the lavas of non-active volcanoes ( 1 group)), and calculated the average compositions individually. The frequency distribution diagrams of each group, based on $\mathrm{SiO}_{2}, 100 \mathrm{MgO} /$ Total $\mathrm{FeO}+\mathrm{MgO}+\mathrm{Alk}$ and $100 \mathrm{Alk} / \mathrm{Total} \mathrm{FeO}+\mathrm{MgO}+\mathrm{Alk}$, were also constructed. The results obtained are as summarized in this paper.

The characteristics of each group in the average chemical compositions and frequency distibution curves of each group seem to be revealing many important problems, which should be discussed by volcanologist, especially standing on the petrological (and geological) viewpoint. 


\section{Lavas of Japanese volcanoes as a whole}

The lavas refer mainly to lava flows, although a small amount of bombs or pumices produced by distinctive eruptions are also included.

As first the frequency distribution and the average chemical compositions of all analysed lavas of Japanese Quaternary volcanoes are given in Table 1 and Fig. 1.

The highest peak of silica percentage appears to be between 57 and $61 \%$, and the second peak between $51-63 \%$, in Fig. 1. However, it should be noticed that the

Table 1. Average chemical compositions of lavas (lava, flow bomb, pumice) of Japanese volcanoes as a whole (The sum of $1,2,3$ and 4 of Table 2)

\begin{tabular}{|c|c|c|c|c|c|c|c|}
\hline No. of anal. & $\begin{array}{c}\text { I } \\
36\end{array}$ & $\underset{145}{\text { II }}$ & $\begin{array}{r}\text { III } \\
189\end{array}$ & $\begin{array}{r}\text { IV } \\
141\end{array}$ & $\begin{array}{l}\mathrm{V} \\
57\end{array}$ & $\begin{array}{l}\text { VI } \\
40\end{array}$ & $\begin{array}{l}\text { VII } \\
11\end{array}$ \\
\hline $\mathrm{SiO}_{2}$ & 49.27 & 52.37 & 57.64 & 62.15 & 67.25 & 72.43 & 76.19 \\
\hline $\mathrm{TiO}_{2}$ & 0.98 & 0.97 & 0.83 & 0.67 & 0.54 & 0.28 & 0.13 \\
\hline $\mathrm{Al}_{2} \mathrm{O}_{3}$ & 18.44 & 17.68 & 17.17 & 16.24 & 15.22 & 14.11 & 12.86 \\
\hline $\mathrm{Fe}_{2} \mathrm{O}_{3}$ & 3.35 & 3.34 & 3.12 & 2.83 & 2.06 & 1.14 & 0.78 \\
\hline $\mathrm{FeO}$ & 7.21 & 7.15 & 4.87 & 3.63 & 2.41 & 1.56 & 0.52 \\
\hline $\mathrm{MnO}$ & 0.17 & 0.17 & 0.15 & 0.13 & 0.09 & 0.08 & 0.06 \\
\hline $\mathrm{MgO}$ & 5.76 & 4.68 & 3.58 & 2.45 & 1.30 & 0.67 & 0.29 \\
\hline $\mathrm{CaO}$ & 11.00 & 9.58 & 7.33 & 5.72 & 4.17 & 2.41 & 1.34 \\
\hline $\mathrm{Na}_{2} \mathrm{O}$ & 2.26 & 2.50 & 2.93 & 3.35 & 3.65 & 3.28 & 4.04 \\
\hline $\mathrm{K}_{2} \mathrm{O}$ & 0.55 & 0.64 & 1.21 & 1.59 & 1.74 & 2.67 & 2.42 \\
\hline $\mathrm{H}_{2} \mathrm{O}+$ & 0.54 & 0.52 & 0.68 & 0.73 & 1.04 & 0.91 & 1.05 \\
\hline $\mathrm{H}_{2} \mathrm{O}-$ & 0.36 & 0.34 & 0.46 & 0.43 & 0.46 & 0.30 & 0.23 \\
\hline $\mathrm{P}_{2} \mathrm{O}_{5}$ & 0.16 & 0.16 & 0.18 & 0.21 & 0.19 & 0.12 & 0.07 \\
\hline Total & 100.05 & 100.09 & 100.15 & 100.13 & 100.12 & 99.96 & 99.98 \\
\hline $\begin{array}{c}\mathrm{Fe}_{2} \mathrm{O}_{3} \\
\mathrm{FeO}\end{array}$ & 0.46 & 0.46 & 0.64 & 0.77 & 0.85 & 0.73 & 1.50 \\
\hline$\frac{\mathrm{Al}_{2} \mathrm{O}_{3}}{\mathrm{CaO}}$ & 1.67 & 1.84 & 2.34 & 2.83 & 3.64 & 5.85 & 9.59 \\
\hline$\frac{\mathrm{Na}_{2} \mathrm{O}}{\mathrm{K}_{2} \mathrm{O}}$ & 4.10 & 3.90 & 2.42 & 2.10 & 2.09 & 1.22 & 1.66 \\
\hline$\frac{\text { Total } \mathrm{FeO}}{\mathrm{MgO}}$ & 1.80 & 2.20 & 2.18 & 2.57 & 3.34 & 3.97 & 4.41 \\
\hline$\frac{\mathrm{Al}_{2} \mathrm{O}_{3}}{\mathrm{Na}_{2} \mathrm{O}+\mathrm{K}_{2} \mathrm{O}}$ & 6.56 & 5.63 & 4.14 & 3.28 & 2.82 & 2.37 & 1.99 \\
\hline Total $\mathrm{FeO}$ & 54.4 & 56.5 & 49.8 & 45.5 & 38.9 & 28.0 & 15.3 \\
\hline $\mathrm{MgO}$ & 30.7 & 26.0 & 23.3 & 18.1 & 11.9 & 7.3 & 3.6 \\
\hline $\mathrm{Na}_{2} \mathrm{O}+\mathrm{K}_{2} \mathrm{O}$ & 15.0 & 17.5 & 26.9 & 36.4 & 49.2 & 64.7 & 81.1 \\
\hline
\end{tabular}


curve seems to be a combination of several symmetrical frequency curves, the principal peaks of each curve being between $\mathrm{SiO}_{2} 51-53,56-58,59-62,63-65$, etc.

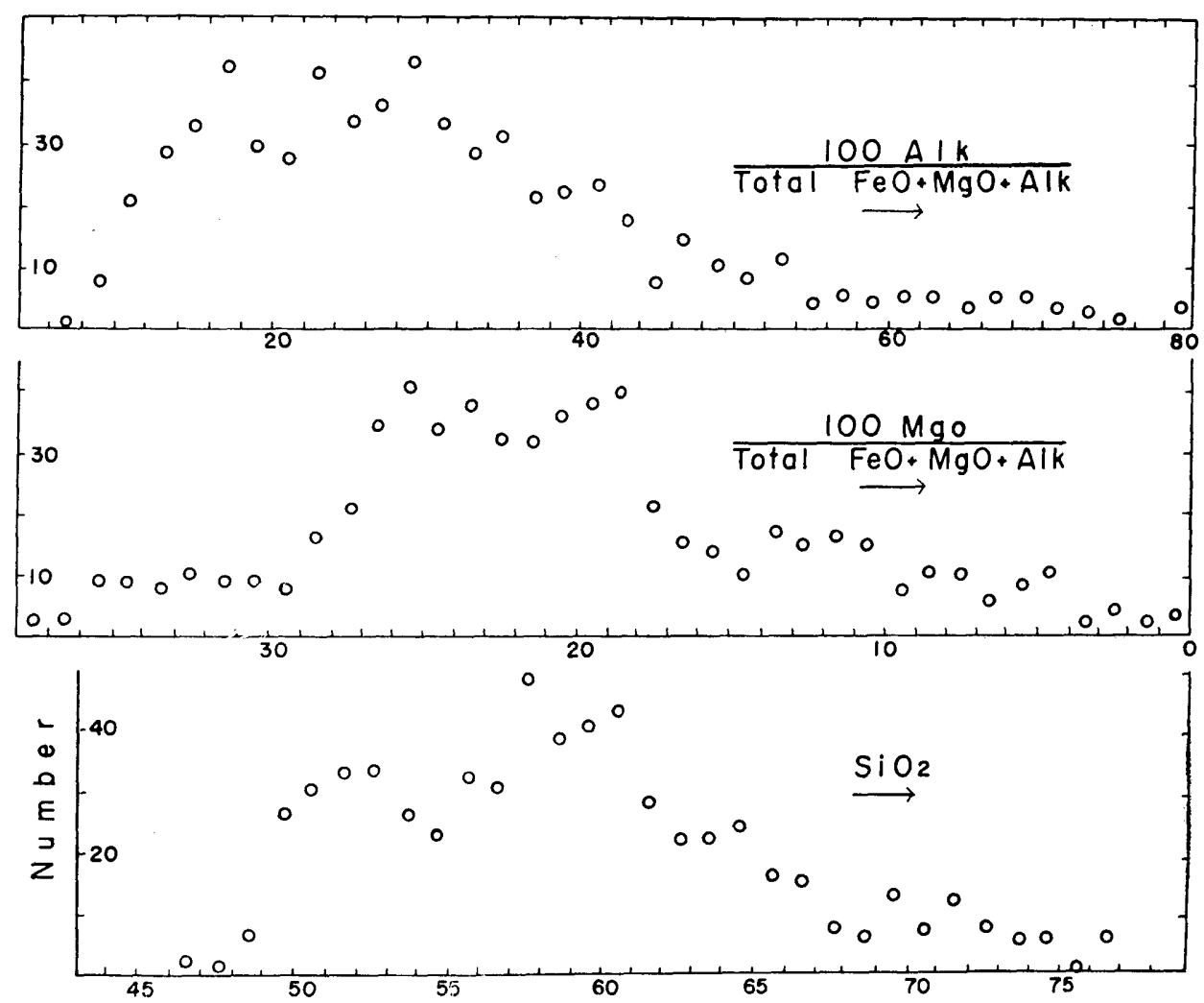

Fig. 1. Frequency distribution diagrams for Japanese volcanic rocks as a whole

The highest peak of $100 \mathrm{MgO} /$ Total $\mathrm{FeO}+\mathrm{MgO}+\mathrm{Alk}$ may be between 18 and 26 (perhaps 18 and 20), and that of $100 \mathrm{Alk} / \mathrm{Total} \mathrm{FeO}+\mathrm{MgO}+\mathrm{Alk}$ between 22 and 30 (perhaps 28 and 30 ). The both curves have the second peaks also.

It should be noticed that the frequency distribution curve show two principal peaks.

The new average chemical compositions hardly differ from the old average (Taneda, 1952), e. g. the alkali-lime index is 63.8 as against the old average of 63.7 .

\section{Grouping of lavas of Japanese volcanoes}

I divided the volcanoes mentioned above into 2 groups: (I) active and (II) nonactive and divided the former into 2 subgroups, (a) volcanoes which have ejected lavas in historic times and (b) volcanoes which are active but have never ejected lavas (lava flow) in historic times. Moreover the lavas of historic times were separated from those of prehistoric times. The grouping of the lavas are as shown in Table 2. 
Table 2 Groupings of the lavas and volcanoes

1 The lavas of historic times

2 The lavas of prehistoric times of the volcanoes $\left\{\begin{array}{c}\text { Active (a) } \\ 1+2\end{array}\right.$

$\left.\begin{array}{c}\text { The lavas of the other active volcanoes which have } \\ \text { never flowed out lava in historic times }\end{array}\right\}$ Active (b) $\} \begin{array}{r}\text { I. Active volcano } \\ 1+2+3\end{array}$

$\left.\begin{array}{l}4 \text { The lavas of the volcanoes with no recorded erup. } \\ \text { tions }\end{array}\right\}$. . . I I. Non-active volcanoes

* A few volcanoes which have ejected considerable amount of bombs or pumices in distinctive eruptions are also treated.

** The older volcanoes constructing the base of active volcanoes, i.e. the older volcano of $\overrightarrow{\mathrm{O}}$-sima, the sommas of Aso, Aira, etc. are also treated.

The classification of volcanoes according to their activity at the present appears not to be geological, but it is not insignificant because we can presume that the group of active volcanoes may be superior to the group of non-active volcanoes in the number of volcanoes which should retain their activity for a long time in the future, even if some of the non-active volcanoes might rejuvenate in the future. It is also somewhat significant to separate the volcanoes (a) which have produced lava flow in historic times from the volcanoes (b) which have exploded but never

Table 3. Volcanoes having analytical data of lavas

1. Active volcanoes, including solfatara fields

(a) Active volcanoes, which have ejected lava (flow) in historic times

A few volcanoes which have ejected considerable amount ef bombs or pumices by the distinct eruptions in historic times, not accompanied by lava flow, are also treated.

Tokati-dake (Tokachi-dake)* Tarumai; Usu; Komaga-dake; Iwate; Tyokai (Chyokai); Asama; Huzi (Fuji); Mihara-yama, Ỏsima (Ōshima); Miyake-sima (Miyake-zima); Torisima, Izu; Iwo-sima (Iwo-zima), Volcanic Islands; Aso*, Unzen, Sakura-zima (Sakura-jima); Iwo-sima (Iwo-zima), Satunan; Suwanose-zima.

* Volcanoes with no recorded lava flows in historic times.

(b) Active volcanoes, which have apparently never ejected lava (flow) in historic times Siretoko Iwo-yama (Shiretoko Iwo-yama); Daisetu*; Atosanupli*; Osima-Ösima (OshimaÖshima); Me-akan; Iwaki, Akita-Yake-yama; Hatimantai*; Akita-Komaga-dake; Kurikoma; Narugo*; ZaO ; Asama ; Adatara; Bandai ; Nasu ; Kusatu-Sirane, Akagi, Hakone*; Ömuroyama*; Nii-zima; Kōzu-sima ; Hatizyo-zima; Ontake»; Kuzyu (Kuju); Kirisima (Kirishima); Kutino-erabu; Nakano-sima

* Volcanoes with no recorded eruptions.

2. Volcanoes with no recorded eruptions (non-active or extinct volcanoes)

Kutzsharo (Kutcharo); Irmukeppu; Masyu (Mashu); Akan; Risiri (Rishiri); Yōtei (Siribesi, Shiribeshi); Niseko; Towada ; Funagata; Kampu; Kassan; Yudono-yama; Nekoma ; Kayōdake; Numazawa; Moriyosi (Moriyoshi); Takahara; Nantai Nyohō.Akanagi; Amagi; Haruna; Taga ; Asitaka ; Usami ; Utone-sima; Mikura-sima ; To-sima ; Iizuna; Kurohime ; Kayagatake ; Sambe; Aono ; Hime-sima ; Hutago; Tara-dake ; Kutino-sima; Taira-sima ; Tokati older bodies (w.t.); Sikotu (w.t.); Noboribetu (w.t.); Tamakawa (w.t.); Aso (w.t.); Aira (w.t.); Ỏ-sima, Izu (o.b.).

Abbr. w.t.-welded tuff, O.b.-Older bodies. 
Literatures including the chemical data of the products of Japanese volcanoes

Tada, H. \& Tsuya, H. (1927)-Bull. Earthq. R.I., 2; Suzuki, J. (1935)-Bull. Vol. S.J., 2; Katsui, Y. \& Takahashi, T. (1960)-J. Jap. As. P.M.E'G., 44; Ishikawa, T. (1952)-J. Fac. Sci. Hokkaido Univ., IV, 1; Katsui, Y. (1952)-Bull. Geol. Com. Hakkaido, No. 38; Sato, D. (1913)-Geol. surv.; Yagi, K. (1953)-Trans. Am. Geoph. Un., 34 ; Sedo, K. (1931)-J. Jap. As. P.M.E'G., 6; Sedo, K. \& Yagi, T. (1931)-J. Jap. As. P.M.E'G., 5; Tsuya, H. (1929)-Bull. Earthq. R.I., 7; Kawano, Y. \& Aoki, K. (1959)-Sc. Rep. Tohoku Univ., III, 4; Katsui, Y. (1954)-J. Geol. S. J., 60 ; Kozu, S. (1932)-Bull. Volc. S. J., 1; Tsuya, H. (1933)-Geog., 2; Iwasaki, I. (1935)-J. Chem. S. J., 56; Tsuya, H. (1937)-Bull. Earthq. R.I., 15; Tsuboi, S. (1917)-J. Geol. S. J., 24; Hsuya, H. \& Morimoto, R. (1951)-Bull. Earthq. R. I., 30; Kuno, H. (1958)-Bull. Volc. S. J., 5; Isshiki, N. (1960)-Expl. Text Geol. Map (Hiyake-zima); Morimoto, R. (1957)-C.G.I., XX; (1960)-Assembly Vol. S. J.; Tanakadate, H. (1940)-J. Geol. S. J., 47; Tsuya, H. (1936)-Bull. Earthq. R. I., 14; Iwasaki, I. (1937)-J. Chem. S. J., 63 ; Kawano, Y. (1933)-J. Jap. As. P.M.E'G., 12; Homma, H. \& Mukae, M. (1938)-Bull. Volc. S. J., 4; Matsumoto, H. (1958)-J. Jap. As. P.M.E'G., 43; Taneda, S. (Aso, unpublished); Homma, H. (1936)-Bull. Volc. S. J., 3; Kurasawa, H. \& Takahasi, K. (1959)-Assembly Geol. S. J.; Yamamoto, T. (1960)-Bull. Volc. S. J., 5; Yamaguchi, K. (Taneda, S. 1952-Guidebook, Kyushu Univ.); Morimoto, R. (1948)-Bull. Earthq. R. I., 26; Taneda, S. \& Morita, J. (1958)-J. Jap. As. P.M.E'G., 42; Tanakadate, H. (1935)-Proc. Imp. Ac. Tokyo, 11; Matsumoto, H. (1954)-Kumamoto J. Sc., BI, 1; (1956) Do., 2; Katsui, Y. (1959)-Bull. Geol. Com. Hokkaido, No. 38; Katsui Y. \& Takahashi, T. (1960)J. Jap. As. P.M.E'G., 44; Katsui, Y. (1958)-Earth Sc., 39; Kawano, Y., Yagi, K. \& Aoki, K. (1961)-Sc. Rep. Tohoku Univ., III, 4; Tsuya, H. (1934)-Bull. Earthq. R. I., 12; Kuno, H. (1958)-Bull. Volc. S. J., 3; Nagasima, K. (1953)-Bull. Fac. Agr. Tokyo Univ., Agr. Tech., 1 ; Asayama, T. (1950)-Sc. Rep. Kyoto Tech. Univ., 4; Isshiki, N. (1959)-Expl. Text Geol. Map (Hatizyo-zima); Samesima, T. (1958)-Ontake; Matsumoto, Y. (1958)-Yuhusan; Sawamura, K. \& Takahashi, K. (1957)-Expl. Text Geol. Map (Kiri-sima); Matsumoto, H. (1960)-Kumamoto J. Sc., BI, 4; Kasama, T. (1959)-Bull. Ôsaka Museum N. H., No. 11; Kawano, Y., Matsui, K. \& Simizu, I. (1956)-Expl. Text Geol. Map (Utashinai); Katsui, Y. (1953)-J. Fac. Sc. Hokkaido Univ., IV, 8; Oba, Y. (1960)-J. geol. S. J., 63; Miyazaki, S. (1937)-Tokyo Univ., M.S. ; Ôhasi, R. (1931)-J. Geol. S. J., 38; Kawano, Y. (1939)-J. Jap. As. P.M.E.G., 22 ; Yamasaki, M. (1954)-J. Fac. Sc. Univ. Tokyo, II, 9; Kurasawa, H. (1859)-Earth Sc., No. 44; Yamasaki, M. (1959)-Bull. Volc. S. J., II, 2; Taneda, S. (Haruna, unpublished); Kuno, H. (1936); Bull. Volc. S. J.; Yamada, S. (1934)-Bull. Earthq. R. I., 12; Ichiki, M. (1929)-Bull. Earthq. R. I., 7; Kawano, Y. (1943)-J. Geog.; Iwasaki, I. \& Katsura, T. (1949)-Assembly Chem. Sc. J.; Haraguchi, K. (1930)-Chikyu, 14; Kawano, Y. (1950)-Rep. No. 134, Geol. Surv. J.; Taneda, S. (Hime-sima, unpublished); Kawano, Y. (1937)-J. Jap. As. P.M.E'G., 18; Takahasi, K. \& Kurasawa, H. (1960)-Bull. Geol. Survey J., 11; Suzuki, T. (1957)-Bull. Geol. Com. Hokkaido, No. 31; Yamaguchi, K. (1937-38)-J. Geol. S. J., 44 \& 45; Kuno, H. (1932)-Bull. Vol. S. J. 1.

Abbr. J : Journal or Japan, P.M.E'G.-Petr. Min. Eco' Geol., R.I.-Research Inst., S.--Society 
produced lava flow in historic times, because it is referable to the grouping of types of volcanic activities.

Table 4. Average (except column V) chemical compositions of lavas (including few bombs \& pumices) of historic times in Japan (1 of Table 2).

\begin{tabular}{|c|c|c|c|c|c|}
\hline No. of anal. & $\underset{20}{I}$ & $\underset{10}{\Pi}$ & $\begin{array}{l}\text { III } \\
12\end{array}$ & $\begin{array}{c}\text { IV } \\
9\end{array}$ & $\begin{array}{l}\mathrm{V} \\
1\end{array}$ \\
\hline $\mathrm{SiO}_{2}$ & 52.56 & 58.53 & 61.73 & 67.61 & 71.25 \\
\hline $\mathrm{TiO}_{2}$ & 1.18 & 0.72 & 0.65 & 0.57 & 0.43 \\
\hline $\mathrm{Al}_{2} \mathrm{O}_{3}$ & 16.47 & 17.17 & 16.18 & 15.23 & 13.21 \\
\hline $\mathrm{Fe}_{2} \mathrm{O}_{3}$ & 3.30 & 2.41 & 2.20 & 1.62 & 3.19 \\
\hline $\mathrm{FeO}$ & 8.50 & 5.38 & 4.32 & 2.88 & 1.96 \\
\hline $\mathrm{MnO}$ & 0.20 & 0.27 & 0.15 & 0.11 & 0.27 \\
\hline $\mathrm{MgO}$ & 4.44 & 3.19 & 2.75 & 1.36 & 0.84 \\
\hline $\mathrm{CaO}$ & 9.65 & 7.66 & 6.11 & 4.36 & 3.10 \\
\hline $\mathrm{Na}_{2} \mathrm{O}$ & 2.40 & 2.87 & 3.49 & 3.68 & 4.02 \\
\hline $\mathrm{K}_{2} \mathrm{O}$ & 0.77 & 1.31 & 1.55 & 1.38 & 1.15 \\
\hline $\mathrm{H}_{2} \mathrm{O}+$ & 0.29 & 0.52 & 0.51 & 0.60 & 0.50 \\
\hline $\mathrm{H}_{2} \mathrm{O}-$ & 0.29 & 0.24 & 0.21 & 0.27 & 0.20 \\
\hline $\mathrm{P}_{2} \mathrm{O}_{5}$ & 0.18 & 0.19 & 0.18 & 0.18 & 0.46 \\
\hline Total & 100.22 & 100.46 & 100.03 & 99.85 & 100.63 \\
\hline$\frac{\mathrm{Al}_{2} \mathrm{O}_{3}}{\mathrm{CaO}}$ & 1.70 & 2.24 & 2.65 & 3.49 & 3.49 \\
\hline$\frac{\mathrm{Na}_{2} \mathrm{O}}{\mathrm{K}_{2} \mathrm{O}}$ & 3.11 & 2.19 & 2.25 & 2.66 & 4.25 \\
\hline$\frac{\text { Total FeO }}{\mathrm{MgO}}$ & 2.62 & 2.44 & 2.36 & 3.26 & 11.10 \\
\hline$\frac{\mathrm{Al}_{2} \mathrm{O}_{3}}{\mathrm{Na} \mathrm{O}+\mathrm{K}_{2} \mathrm{O}}$ & 3.19 & 4.10 & 3.21 & 3.00 & 2.55 \\
\hline$\frac{\mathrm{Fe}_{2} \mathrm{O}_{3}}{\mathrm{FeO}}$ & 0.38 & 0.44 & 0.46 & 0.56 & 0.31 \\
\hline Total FeO & 60.1 & 50.6 & 44.67 & 40.3 & 44.6 \\
\hline $\mathrm{MgO}$ & 23.3 & 21.4 & 19.5 & 12.7 & 7.7 \\
\hline $\mathrm{Na}_{2} \mathrm{O}+\mathrm{K}_{2} \mathrm{O}$ & 16.6 & 28.0 & 35.8 & 47.1 & 47.6 \\
\hline
\end{tabular}

I $\quad 50 \leqq \mathrm{SiO}_{2}<55, \quad$ II $55 \leqq \mathrm{SiO}_{2}<60, \quad$ III $60 \leqq \mathrm{SiO}_{2} 65 \quad$ IV $65 \leqq \mathrm{SiO}_{2}<70$ 
Table 5. Average chemical compositions of lavas (lava flow, bomb, pumice) of prehistoric times of Japanese active volcanoes which have ejected lava (flow) in historic times (2 of Table 2)

\begin{tabular}{|c|c|c|c|c|c|c|}
\hline No. of anal. & $\begin{array}{l}I \\
7\end{array}$ & $\frac{\text { II }}{25}$ & $\begin{array}{l}\text { III } \\
19\end{array}$ & $\begin{array}{l}\text { IV } \\
20\end{array}$ & $\begin{array}{l}V \\
6\end{array}$ & $\begin{array}{r}\mathrm{VI} \\
3\end{array}$ \\
\hline $\mathrm{SiO}_{2}$ & 49.21 & 52.23 & 58.11 & 61.40 & 66.11 & 71.71 \\
\hline $\mathrm{TiO}_{2}$ & 1.02 & 1.07 & 0.70 & 0.72 & 0.59 & 0.54 \\
\hline $\mathrm{Al}_{2} \mathrm{O}_{3}$ & 17.89 & 17.59 & 17.00 & 16.61 & 15.24 & 14.15 \\
\hline $\mathrm{Fe}_{2} \mathrm{O}_{3}$ & 3.84 & 3.42 & 3.00 & 2.98 & 1.87 & 0.93 \\
\hline $\mathrm{MnO}$ & 0.18 & 0.20 & 0.19 & 0.13 & 0.09 & 0.11 \\
\hline $\mathrm{MgO}$ & 5.29 & 4.40 & 3.36 & 2.76 & 1.40 & 0.29 \\
\hline $\mathrm{CaO}$ & 10.62 & 9.53 & 7.03 & 5.96 & 4.43 & 2.18 \\
\hline $\mathrm{Na}_{2} \mathrm{O}$ & 2.06 & 2.61 & 3.10 & 3.11 & 3.66 & 4.25 \\
\hline $\mathrm{K}_{2} \mathrm{O}$ & 0.60 & 0.72 & 1.46 & 1.81 & 2.11 & 3.13 \\
\hline $\mathrm{H}_{2} \mathrm{O}+$ & 0.50 & 0.49 & 0.64 & 0.71 & 0.63 & 0.87 \\
\hline $\mathrm{H}_{2} \mathrm{O}-$ & 0.24 & 0.26 & 0.40 & 0.51 & 0.54 & 0.40 \\
\hline $\mathrm{P}_{2} \mathrm{O}_{5}$ & 0.17 & 0.18 & 0.20 & 0.17 & 0.21 & 0.07 \\
\hline Total & 99.90 & 100.18 & 100.14 & 100.27 & 100.24 & 100.01 \\
\hline$\frac{\mathrm{Al}_{2} \mathrm{O}_{3}}{\mathrm{CaO}}$ & 1.68 & 1.84 & 2.41 & 2.78 & 3.44 & 6.49 \\
\hline$\frac{\mathrm{Na}_{2} \mathrm{O}}{\mathrm{K}_{2} \mathrm{O}}$ & 3.43 & 3.62 & 2.12 & 1.71 & 1.73 & 1.35 \\
\hline$\frac{\text { Total } \mathrm{FeO}}{\mathrm{MgO}}$ & 2.25 & 2.42 & 2.32 & 2.28 & 3.66 & 8.34 \\
\hline$\frac{\mathrm{Al}_{2} \mathrm{O}_{3}}{\mathrm{Na} \mathrm{a}_{2} \mathrm{O}+\overline{\mathrm{K}}_{2} \mathrm{O}}$ & 6.72 & 5.28 & 3.72 & 3.37 & 2.64 & 1.91 \\
\hline$\frac{\mathrm{Fe}_{2} \mathrm{O}_{3}}{\mathrm{FeO}}$ & 0.46 & 0.46 & 0.60 & 0.85 & 0.55 & 0.67 \\
\hline Toal $\mathrm{FeO}$ & 59.5 & 57.5 & 49.1 & 44.6 & 41.3 & 23.2 \\
\hline $\mathrm{MgO}$ & 26.9 & 24.2 & 21.6 & 19.9 & 11.5 & 2.9 \\
\hline $\mathrm{Na}_{2} \mathrm{O}+\mathrm{K}_{2} \mathrm{O}$ & 13.5 & 18.3 & 29.3 & 35.5 & 47.3 & 73.9 \\
\hline
\end{tabular}

I $\mathrm{SiO}_{2}<50 \%, \quad$ II $50 \leqq \mathrm{SiO}_{2}<55$, III $55 \leqq \mathrm{SiO}_{2}<60, \quad$ IV $60 \leqq \mathrm{SiO}_{2}<65, \quad$ V $65 \leqq \mathrm{SiO}_{2}<70$, VI $70 \leq \mathrm{SiO}_{2}<75$ 
Table 6. Average chemical compositions of lavs (lava flow, bomb, pumice) of Japanese active volcanoes which have never flowed out lavas in historic times ( 3 of Table 2)

\begin{tabular}{|c|c|c|c|c|c|c|c|}
\hline No. of anal. & $\begin{array}{c}\mathrm{I} \\
12\end{array}$ & $\begin{array}{l}\text { II } \\
32\end{array}$ & $\frac{\text { III }}{86}$ & $\begin{array}{l}\text { IV } \\
35\end{array}$ & $\begin{array}{c}\mathrm{V} \\
14\end{array}$ & $\begin{array}{l}\text { VI } \\
12\end{array}$ & VII \\
\hline $\mathrm{SiO}_{2}$ & 49.33 & 52.64 & 57.49 & 62.08 & 67.74 & 71.87 & 75.96 \\
\hline $\mathrm{TiO}_{2}$ & 0.99 & 0.98 & 0.90 & 0.70 & 0.68 & 0.34 & 0.11 \\
\hline $\mathrm{Al}_{2} \mathrm{O}_{3}$ & 18.49 & 17.60 & 16.90 & 16.04 & 14.99 & 14.49 & 13.31 \\
\hline $\mathrm{Fe}_{2} \mathrm{O}_{3}$ & 3.40 & 3.13 & 3.07 & 2.68 & 2.23 & 1.12 & 0.59 \\
\hline $\mathrm{FeO}$ & 7.58 & 7.23 & 5.22 & 4.21 & 2.30 & 1.71 & 0.48 \\
\hline $\mathrm{MnO}$ & 0.19 & 0.16 & 0.14 & 0.12 & 0.08 & 0.06 & 0.08 \\
\hline $\mathrm{MgO}$ & 5.43 & 4.89 & 3.78 & 2.60 & 1.18 & 0.82 & 0.27 \\
\hline $\mathrm{CaO}$ & 11.05 & 9.64 & 7.49 & 5.91 & 3.83 & 2.67 & 1.15 \\
\hline $\mathrm{Na}_{2} \mathrm{O}$ & 1.98 & 2.32 & 2.76 & 3.19 & 3.69 & 3.07 & 4.25 \\
\hline $\mathrm{K}_{2} \mathrm{O}$ & 0.58 & 0.56 & 1.15 & 1.57 & 1.51 & 2.86 & 2.14 \\
\hline $\mathrm{H}_{2} \mathrm{O}+$ & 0.61 & 0.50 & 0.67 & 0.60 & 1.08 & 0.94 & 1.43 \\
\hline $\mathrm{H}_{2} \mathrm{O}-$ & 0.30 & 0.32 & 0.42 & 0.39 & 0.57 & 0.24 & 0.10 \\
\hline $\mathrm{P}_{2} \mathrm{O}_{5}$ & 0.16 & 0.10 & 0.14 & 0.16 & 0.17 & 0.11 & 0.05 \\
\hline Total & 100.09 & 100.08 & 100.14 & 100.25 & 100.05 & 100.30 & 99.92 \\
\hline $\begin{array}{c}\mathrm{Fe}_{2} \mathrm{O}_{3} \\
\mathrm{FeO}\end{array}$ & 0.44 & 0.43 & 0.58 & 0.63 & 0.96 & 0.65 & 1.22 \\
\hline$\frac{\mathrm{Al}_{2} \mathrm{O}_{3}}{\mathrm{CaO}}$ & 1.67 & 1.82 & 2.25 & 2.71 & 3.91 & 5.42 & 11.57 \\
\hline$\frac{\mathrm{Na}_{2} \mathrm{O}}{\mathrm{K}_{2} \mathrm{O}}$ & 3.41 & 4.14 & 2.40 & 2.04 & 2.44 & 1.07 & 1.98 \\
\hline$\frac{\text { Total } \mathrm{FeO}}{\mathrm{MgO}}$ & 1.99 & 2.08 & 2.14 & 2.58 & 3.72 & 3.37 & 4.03 \\
\hline$\frac{\mathrm{Al}_{2} \mathrm{O}_{3}}{\mathrm{Na}_{2} \mathrm{O}+\mathrm{K}_{2} \mathrm{O}}$ & 7.33 & 6.11 & 4.32 & 3.36 & 2.88 & 2.44 & 2.08 \\
\hline Total $\mathrm{FeO}$ & 57.1 & 56.4 & 50.9 & 47.2 & 40.3 & 28.6 & 13.2 \\
\hline $\mathrm{MgO}$ & 29.1 & 27.4 & 24.1 & 18.6 & 11.0 & 8.7 & 3.5 \\
\hline $\mathrm{Na} a_{2} \mathrm{O}+\mathrm{K}_{2} \mathrm{O}$ & 13.7 & 16.1 & 25.0 & 34.2 & 48.6 & 62.7 & 83.3 \\
\hline
\end{tabular}

I $\mathrm{SiO}_{2}<50 \%, \quad$ II $50 \leqq \mathrm{SiO}_{2}<55, \quad$ III $55 \leqq \mathrm{SiO}_{2}<60, \quad$ IV $60 \leqq \mathrm{SiO}_{2}<65, \quad$ V $65 \leqq \mathrm{SiO}_{2}<70$, VI $70 \leqq \mathrm{SiO}_{2}<75$, VII $75 \leqq \mathrm{SiO}_{2}$ 
Table 7. Average chemical compositions of lavas (lava flow, bomb, pumice) of Japanese volcanoes with no recorded eruptions (4 of Table 2)

\begin{tabular}{|c|c|c|c|c|c|c|c|}
\hline No. of anal. & $\underset{17}{I}$ & $\underset{68}{\text { II }}$ & $\begin{array}{l}\text { III } \\
74\end{array}$ & $\begin{array}{l}\text { IV } \\
74\end{array}$ & $\begin{array}{c}\mathrm{V} \\
28\end{array}$ & $\begin{array}{l}\mathrm{VI} \\
24\end{array}$ & $\begin{array}{r}\text { VII } \\
6\end{array}$ \\
\hline $\mathrm{SiO}_{2}$ & 49.25 & 52.24 & 57.58 & 62.42 & 67.13 & 72.79 & 76.38 \\
\hline $\mathrm{TiO}_{2}$ & 0.95 & 0.85 & 0.78 & 0.66 & 0.46 & 0.23 & 0.14 \\
\hline $\mathrm{Al}_{2} \mathrm{O}_{3}$ & 18.65 & 18.11 & 17.54 & 16.24 & 15.33 & 13.91 & 12.48 \\
\hline $\mathrm{Fe}_{2} \mathrm{O}_{3}$ & 3.11 & 3.42 & 3.30 & 2.94 & 2.15 & 1.17 & 0.93 \\
\hline $\mathrm{FeO}$ & 6.51 & 6.61 & 4.37 & 3.30 & 2.11 & 1.34 & 0.56 \\
\hline $\mathrm{MnO}$ & 0.16 & 0.16 & 0.13 & 0.13 & 0.09 & 0.08 & 0.04 \\
\hline $\mathrm{MgO}$ & 6.20 & 4.72 & 3.47 & 2.28 & 1.31 & 0.65 & 0.31 \\
\hline $\mathrm{CaO}$ & 11.12 & 9.78 & 7.15 & 5.53 & 4.23 & 2.31 & 1.50 \\
\hline $\mathrm{Na}_{2} \mathrm{O}$ & 2.54 & 2.60 & 3.10 & 3.51 & 3.62 & 3.63 & 3.87 \\
\hline $\mathrm{K}_{2} \mathrm{O}$ & 0.50 & 0.60 & 1.20 & 1.54 & 1.89 & 2.52 & 2.66 \\
\hline $\mathrm{H}_{2} \mathrm{O}+$ & 0.51 & 0.60 & 0.71 & 0.86 & 1.28 & 0.91 & 0.74 \\
\hline $\mathrm{H}_{2} \mathrm{O}-$ & 0.46 & 0.39 & 0.54 & 0.45 & 0.46 & 0.31 & 0.34 \\
\hline $\mathrm{P}_{2} \mathrm{O}_{5}$ & 0.16 & 0.16 & 0.20 & 0.26 & 0.24 & 0.13 & 0.08 \\
\hline Total & 100.12 & 100.24 & 100.07 & 100.12 & 100.27 & 99.98 & 100.03 \\
\hline$\frac{\mathrm{Fe}_{2} \mathrm{O}_{3}}{\mathrm{FeO}}$ & 0.47 & 0.51 & 0.75 & 0.89 & 1.01 & 0.87 & 1.66 \\
\hline$\frac{\mathrm{Al}_{2} \mathrm{O}_{3}}{\mathrm{CaO}}$ & 1.67 & 1.85 & 2.45 & 2.93 & 3.62 & 6.02 & 8.32 \\
\hline$\frac{\mathrm{Na}_{2} \mathrm{O}}{\mathrm{K}_{2} \mathrm{O}}$ & 5.08 & 4.33 & 2.58 & 2.27 & 1.91 & 1.44 & 1.45 \\
\hline$\frac{\text { Total FeO }}{\mathrm{MgO}}$ & 1.52 & 2.09 & 2.15 & 2.66 & 3.15 & 3.80 & 4.61 \\
\hline$\frac{\mathrm{Al}_{2} \mathrm{O}_{3}}{\mathrm{Na}_{2} \mathrm{O}+\mathrm{K}_{2} \mathrm{O}}$ & 6.13 & 5.65 & 4.07 & 3.21 & 2.78 & 2.26 & 1.91 \\
\hline Toal $\mathrm{FeO}$ & 50.2 & 55.0 & 48.6 & 44.8 & 37.2 & 26.0 & 16.9 \\
\hline $\mathrm{MgO}$ & 33.4 & 26.8 & 23.0 & 17.2 & 12.1 & 7.1 & 3.8 \\
\hline $\mathrm{Na}_{2} \mathrm{O}+\mathrm{K}_{2} \mathrm{O}$ & 16.4 & 18.2 & 28.5 & 38.1 & 50.7 & 66.9 & 79.3 \\
\hline
\end{tabular}

\section{Lavas of each group}

The average Chemical compositions of the lavas of each groups (Table 2) are calculated and listed in tables (Tables 4, 5, 6, and 7). The variation diagrams are constructed as shown in Fig. 5.

The curves of frequency distribution based on the amount of the silica per- 
centages, the ratios $100 \mathrm{Alk} / \mathrm{Total} \mathrm{FeO}+\mathrm{MgO}+\mathrm{Alk}$ and $100 \mathrm{MgO} / \mathrm{Total} \mathrm{FeO}+\mathrm{MgO}+$ Alk. of analysed lavas are given in Figs. 2, 3 and 4 respectively.
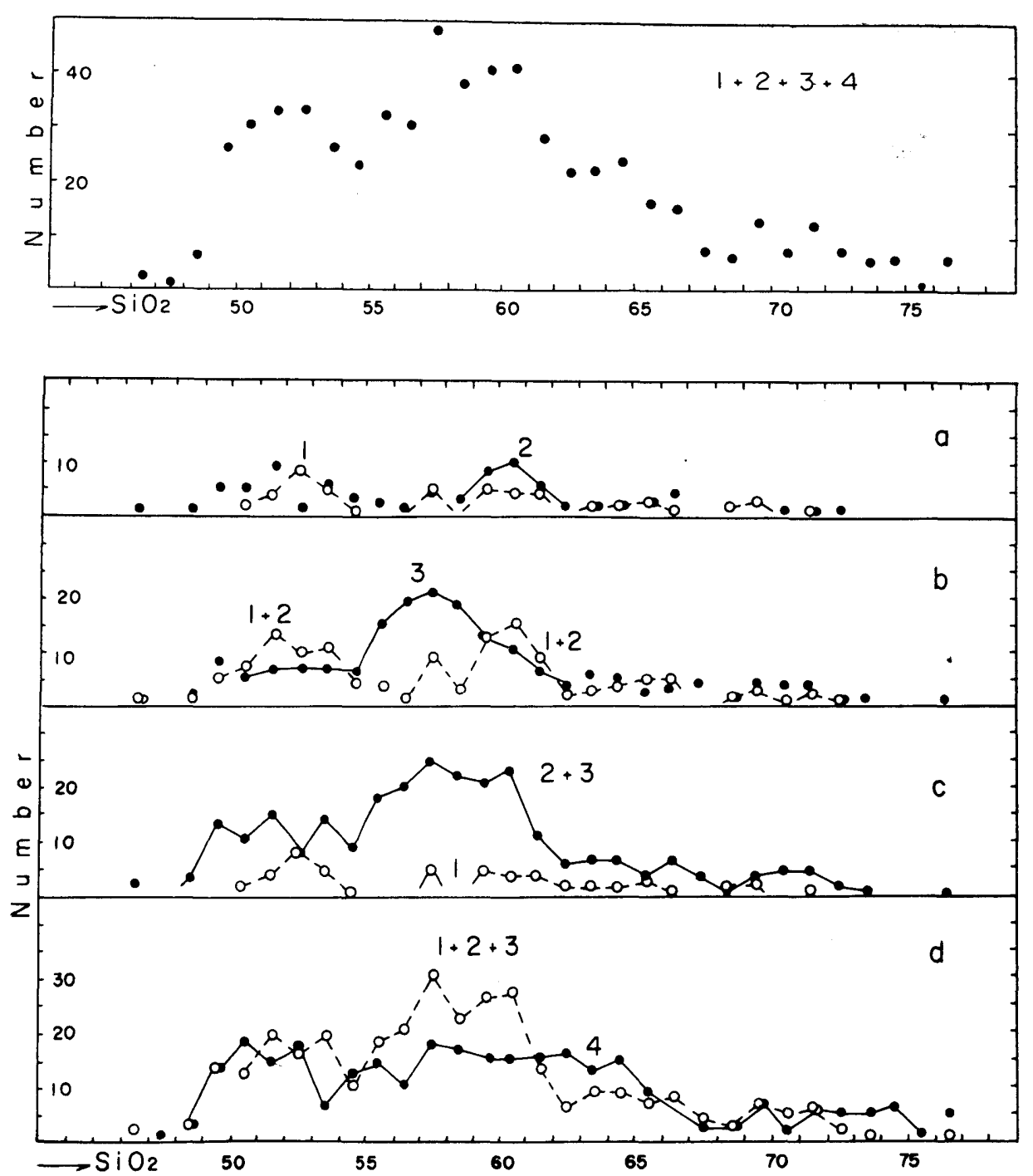

Fig. 2-A. Frequency distribution diagrams. Nos. refer to Table 2.

a Comparison of the lavas of historic times (1) and the lavas of prehistoric times (2).

b Comparison of the lavas of the active volcanoes which have ejected lavas (flow) in historic times $(1+2)$, and the lavas of the other active volcanoes which have never flowed out lavas in historic times (3).

c Comparison of the lavas of historic times (1) and the lavas of prehistoric times of all active volcanoes $(2+3)$.

d Comparison of all lavas of active volcanoes $(1+2+3)$ and all lavas of non-active volcanoes (4). 

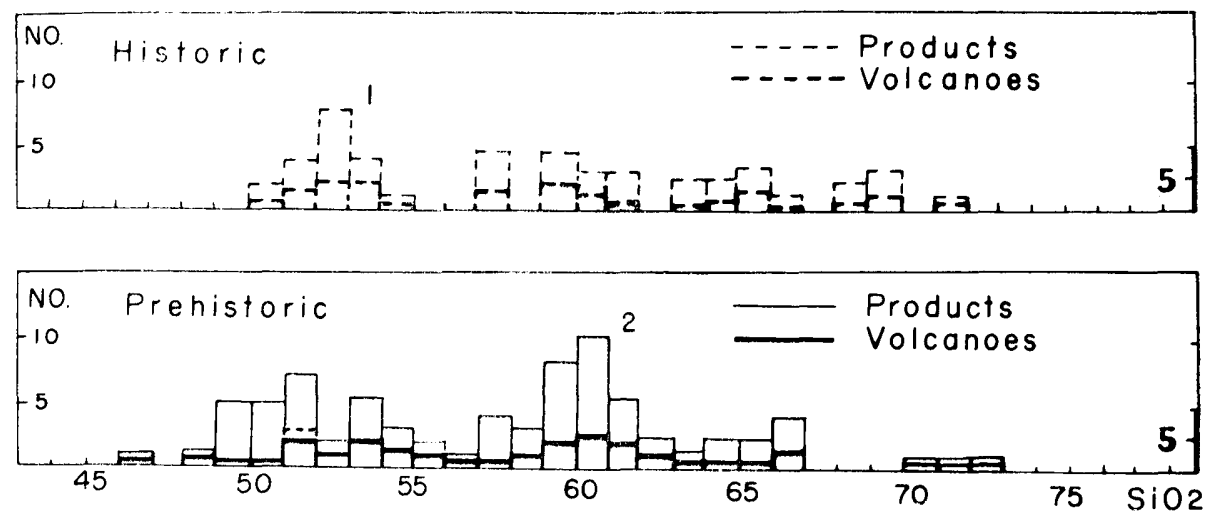

Fig. 2-B. Frequency distribution. 1 \& 2 refer to Fig. 2-A.
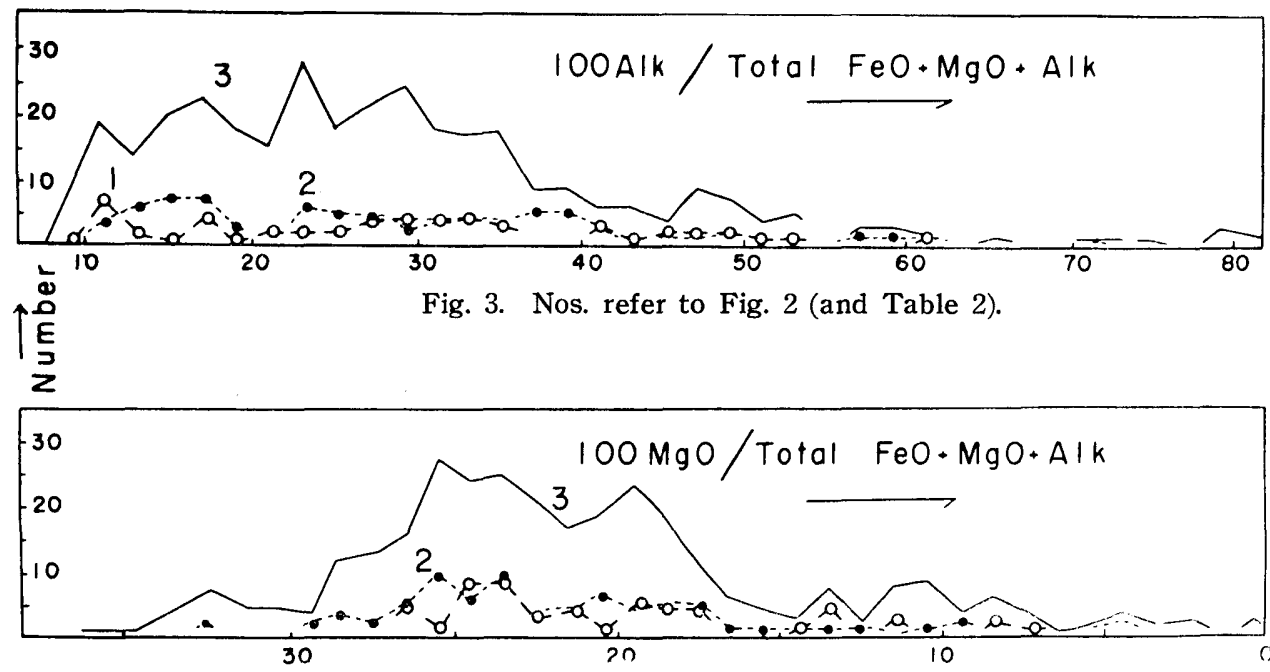

Fig. 4. Nos. refer to Fig. 2 (and Table 2).

\section{Comparison of lavas of historic times and those of prehistoric times}

Compared with the lavas of prehistoric times ( 2 of Table 2), the lavas of historic times ( 1 of Table 2) are characterised by low $\mathrm{K}_{2} \mathrm{O}, \mathrm{H}_{2} \mathrm{O}(+), \mathrm{Fe}_{2} \mathrm{O}_{3} / \mathrm{FeO}$ and high Total $\mathrm{FeO}$ (distinct at $\mathrm{SiO}_{2}<60$ and $>70$ ), $\mathrm{MnO}$ (distinct between $\mathrm{SiO}_{2} 55-60$ ), $\mathrm{CaO}, \mathrm{Na}_{2} \mathrm{O}$ between $\left.\mathrm{SiO}_{2} 60-65\right), \mathrm{Na}_{2} \mathrm{O} / \mathrm{K}_{2} \mathrm{O}\left(\mathrm{SiO}_{2}>55\right), \mathrm{Al}_{2} \mathrm{O}_{3} / \mathrm{Na}_{2} \mathrm{O}+\mathrm{K}_{2} \mathrm{O}\left(\mathrm{SiO}_{2}<60\right.$ and $\left.>65\right)$, Total $\mathrm{FeO} / \mathrm{MgO}\left(\mathrm{SiO}_{2}<65\right.$ and $\left.>70\right)$.

Alkali-lime index is $64.4\left(\mathrm{CaO}=\mathrm{Na}_{2} \mathrm{O}+\mathrm{K}_{2} \mathrm{O}=5.2\right)$ for the lavas of historic times ( 1 of Table 2$)$, and $63.6(\mathrm{CaO}=5.1)$ for those of prehistoric times ( 2 in Table 2).

Concerning the curves of frequency distribution it is noticed that the highest peak of silica porcentage appears to be between $52-53 \%$, and the second peak between $59-62 \%$ for the lavas of historic times (1), though the highest peak appears 


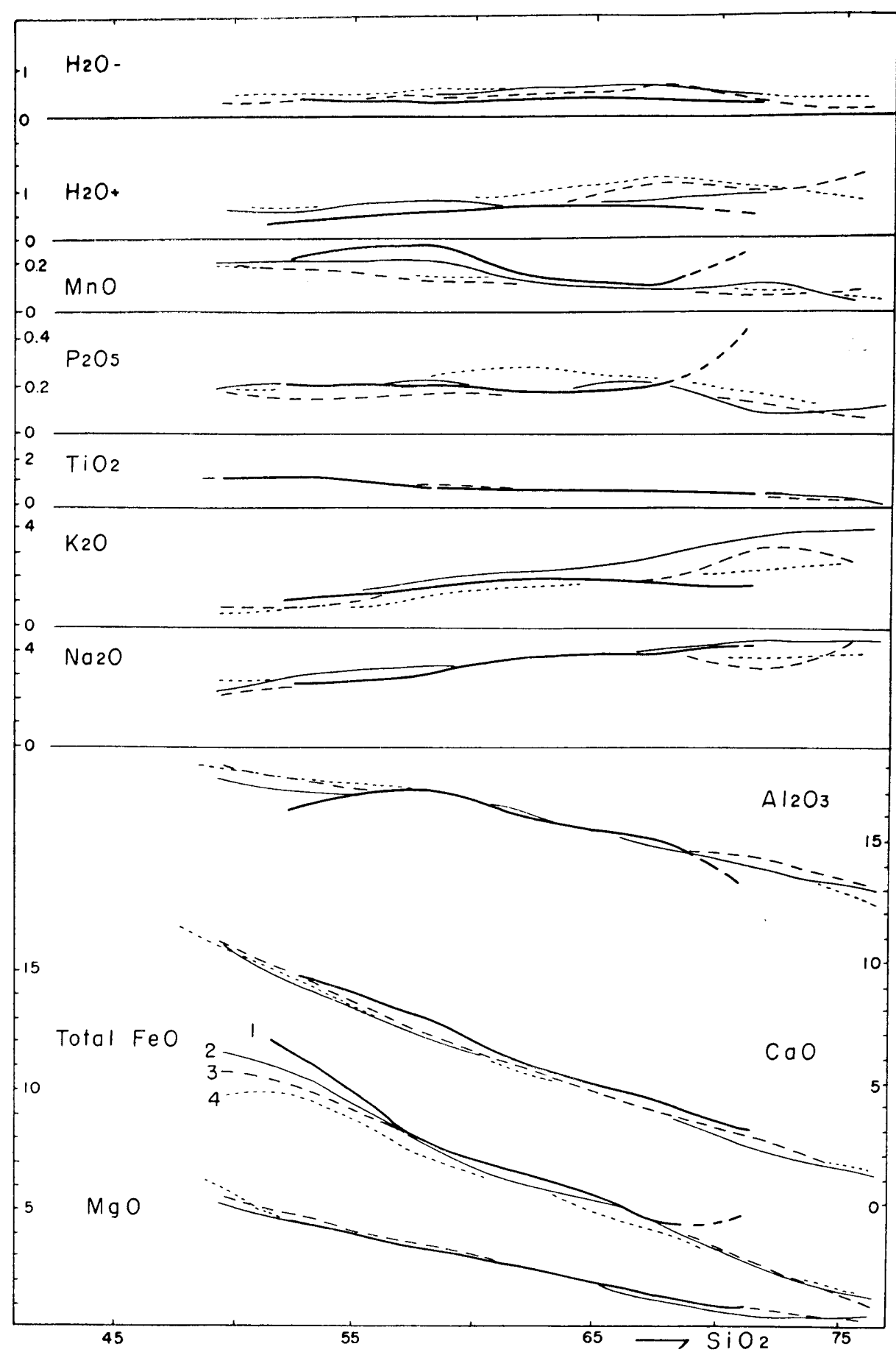

Fig. 5-A. Variation diagram. Nos. refer to Table 2. 
Frequency Distribution and Average Composition of the Volcanic Rocks in Japan

Table 8. Average chemical compositions of lavas (lava flow, bomb, pumice) of active volcanoes in Japan (The sum of 1,2 and 3 of Table 2)

\begin{tabular}{|c|c|c|c|c|c|c|c|}
\hline No. of anal. & $\begin{array}{c}\text { I } \\
19\end{array}$ & $\begin{array}{l}\text { II } \\
77\end{array}$ & III & $\begin{array}{l}\text { IV } \\
67\end{array}$ & $\begin{array}{c}\mathrm{V} \\
29\end{array}$ & $\begin{array}{l}\text { IV } \\
16\end{array}$ & $\begin{array}{l}\text { VII } \\
5\end{array}$ \\
\hline $\mathrm{SiO}_{2}$ & 49.29 & 52.49 & 57.68 & 61.84 & 67.36 & 71.84 & 75.96 \\
\hline $\mathrm{TiO}_{2}$ & 1.00 & 1.07 & 0.86 & 0.69 & 0.63 & 0.27 & 0.11 \\
\hline $\mathrm{Al}_{2} \mathrm{O}_{3}$ & 18.26 & 17.29 & 16.94 & 16.23 & 15.12 & 14.43 & 13.31 \\
\hline $\mathrm{Fe}_{2} \mathrm{O}_{3}$ & 3.56 & 3.26 & 3.00 & 2.71 & 1.97 & 1.08 & 0.59 \\
\hline $\mathrm{FeO}$ & 7.84 & 7.62 & 5.19 & 4.01 & 2.70 & 1.92 & 0.48 \\
\hline $\mathrm{MnO}$ & 0.18 & 0.19 & 0.16 & 0.13 & 0.09 & 0.08 & 0.08 \\
\hline $\mathrm{MgO}$ & 5.38 & 4.62 & 3.65 & 2.64 & 1.29 & 0.72 & 0.27 \\
\hline $\mathrm{CaO}$ & 10.89 & 9.67 & 7.44 & 5.93 & 4.12 & 2.57 & 1.15 \\
\hline $\mathrm{Na}_{2} \mathrm{O}$ & 2.01 & 2.43 & 2.83 & 3.17 & 3.68 & 3.31 & 4.25 \\
\hline $\mathrm{K}_{2} \mathrm{O}$ & 0.59 & 0.67 & 1.22 & 1.65 & 1.60 & 2.92 & 2.14 \\
\hline $\mathrm{H}_{2} \mathrm{O}+$ & 0.57 & 0.44 & 0.66 & 0.60 & 0.83 & 0.92 & 1.43 \\
\hline $\mathrm{H}_{2} \mathrm{O}-$ & 0.28 & 0.30 & 0.41 & 0.41 & 0.46 & 0.29 & 0.10 \\
\hline $\mathrm{P}_{2} \mathrm{O}_{5}$ & 0.17 & 0.16 & 0.16 & 0.16 & 0.18 & 0.10 & 0.05 \\
\hline Total & 100.02 & 100.14 & 100.20 & 100.17 & 100.03 & 100.45 & 99.92 \\
\hline$\frac{\mathrm{Fe}_{2} \mathrm{O}_{3}}{\mathrm{FeO}}$ & 0.45 & 0.42 & 0.57 & 0.67 & 0.72 & 0.56 & 1.22 \\
\hline $\begin{array}{c}\mathrm{Al}_{2} \mathrm{O}_{3} \\
\mathrm{CaO}\end{array}$ & 1.67 & 1.80 & 2.27 & 2.73 & 3.66 & 5.61 & 1.15 \\
\hline$\frac{\mathrm{Na}_{2} \mathrm{O}}{\mathrm{K}_{2} \mathrm{O}}$ & 3.40 & 3.62 & 2.31 & 1.92 & 2.30 & 1.13 & 1.98 \\
\hline$\frac{\text { Total } \mathrm{FeO}}{\mathrm{MgO}}$ & 2.08 & 2.32 & 2.20 & 2.48 & 3.53 & 4.12 & 4.03 \\
\hline$\frac{\mathrm{Al}_{2} \mathrm{O}_{3}}{\mathrm{Na}_{2} \mathrm{O}+\mathrm{K}_{2} \mathrm{O}}$ & 7.02 & 5.57 & 4.18 & 3.36 & 2.86 & 2.31 & 2.08 \\
\hline Toal FeO & 58.0 & 57.7 & 50.6 & 46.3 & 40.5 & 29.4 & 13.2 \\
\hline $\mathrm{MgO}$ & 28.3 & 25.3 & 23.4 & 19.0 & 11.7 & 7.4 & 3.5 \\
\hline $\mathrm{Na}_{2} \mathrm{O}+\mathrm{K}_{2} \mathrm{O}$ & 13.7 & 16.9 & 26.0 & 34.7 & 47.8 & 63.3 & 83.3 \\
\hline
\end{tabular}

I $\mathrm{SiO}_{2}<50 \%, \quad$ II $50 \leqslant \mathrm{SiO}_{2}<55, \quad$ III $55 \leqslant \mathrm{SiO}_{2}<60, \quad$ IV $60 \leqslant \mathrm{SiO}_{2}<65$,

V $65 \leq \mathrm{SiO}_{2}<70$, VI $70 \leq \mathrm{SiO}_{2}<75$, VII $75 \leq \mathrm{SiO}_{2}$ 
Table 9. Comparison of lavas (lava flow, bomb, pumice) of historic times ( $h$ ) with those of prehistoric times $(p h)$ in Eastern and South-eastern Asia.

\begin{tabular}{|c|c|c|c|c|}
\hline Area & $\begin{array}{l}\text { Kamchatka } \\
\text { Active } \\
\text { volcanoes }\end{array}$ & $\begin{array}{c}\text { Kurile } \\
\text { Active } \\
\text { volcanoes }\end{array}$ & $\begin{array}{c}\text { Japan } \\
\text { Active } \\
\text { volcanoes }\end{array}$ & $\begin{array}{l}\text { Indonesia } \\
\text { Active } \\
\text { volcanoes }\end{array}$ \\
\hline Total $\mathrm{FeO}$ & $\mathrm{h}>\mathrm{ph}$ & $\mathrm{h} \geq ? \mathrm{ph}$ & $\begin{array}{c}h>\text { ph } \\
\text { distinct for } \mathrm{SiO}_{2} \\
<60,>70\end{array}$ & $\mathrm{~h}>\mathrm{ph}$ \\
\hline $\mathrm{MnO}$ & $\begin{array}{c}< \\
\mathrm{SiO}_{2} 55-65\end{array}$ & $\begin{array}{c}< \\
\mathrm{SiO}_{2} \quad 55-60\end{array}$ & $\begin{array}{c}> \\
\text { distinct for } \mathrm{SiO}_{2} \\
50-60\end{array}$ & $?$ \\
\hline $\mathrm{CaO}$ & $>$ & $\fallingdotseq$ & $>$ & $\fallingdotseq$ \\
\hline $\mathrm{Na}_{2} \mathrm{O}$ & $\fallingdotseq$ & $>$ & $\begin{array}{c}> \\
\mathrm{SiO}_{2} 60-66\end{array}$ & $\because \because$ \\
\hline $\mathrm{K}_{2} \mathrm{O}$ & $\therefore=$ & $\supseteq$ & $<$ & $<$ \\
\hline $\mathrm{TiO}_{2}$ & $\fallingdotseq$ & $<$ & $\fallingdotseq$ & $<$ \\
\hline $\mathrm{H}_{2} \mathrm{O}(-)$ & $<$ & $<$ & $<$ & $<$ \\
\hline $\mathrm{H}_{2} \mathrm{O}(+)$ & $<$ & $<$ & $<$ & $<$ \\
\hline $\mathrm{Fe}_{2} \mathrm{O}_{3} / \mathrm{FeO}$ & $<$ & $\doteq \bar{Y}$ & $<$ & $<$ \\
\hline $\mathrm{Al}_{2} \mathrm{O}_{3} / \mathrm{CaO}$ & $<?$ & $\because$ & $\leq$ & $\leq ?$ \\
\hline $\mathrm{Na}_{2} \mathrm{O} / \mathrm{K}_{2} \mathrm{O}$ & $\fallingdotseq$ & $>?$ & $\begin{array}{c}> \\
\mathrm{SiO}_{2}>55\end{array}$ & $>$ \\
\hline$\frac{\text { Total FeO }}{\mathrm{MgO}}$ & $?$ & $?$ & $\begin{aligned}> & \\
\mathrm{SiO}_{2} & <65 \\
& >70\end{aligned}$ & $>$ \\
\hline $\mathrm{Al}_{2} \mathrm{O}_{3} / \mathrm{Na}_{2} \mathrm{O}+\mathrm{K}_{2} \mathrm{O}$ & $?$ & $<$ & $\begin{aligned}> & \\
\mathrm{SiO}_{2} & <60 \\
& >65\end{aligned}$ & $\begin{aligned} & > \\
\mathrm{SiO}_{2} & <55 \\
& >65\end{aligned}$ \\
\hline Alkali-lime index & $\begin{array}{cc}\mathrm{h} & \mathrm{h}+\mathrm{ph} \\
62.8>62.2 \\
(5.4) \quad(5.4)\end{array}$ & $\begin{array}{c}\mathrm{h} \quad \mathrm{h}+\mathrm{ph} \\
64.9 \div 64.8 \\
(5.0) \quad(4.9)\end{array}$ & $\begin{array}{cc}\mathrm{h} & \mathrm{ph} \\
64.4> & 63.6 \\
(5.2) & (5.1)\end{array}$ & $\begin{array}{c}\mathrm{h} \quad \mathrm{h}+\mathrm{ph} \\
60.1 \div 60.0 \\
(6.2) \quad(6.2)\end{array}$ \\
\hline $\begin{array}{c}\text { Peak of freq. } \\
\text { curve }\end{array}$ & 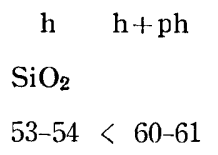 & $\begin{array}{c}\text { h } \quad h+p h \\
\mathrm{SiO}_{2} \\
50-51<(53-63) ?\end{array}$ & $\begin{array}{c}\text { h } \quad h+p h \\
\mathrm{SiO}_{2} \\
52-53<59-61\end{array}$ & $\begin{array}{l}\text { h } \quad h+p h \\
\mathrm{SiO}_{2} \\
54-55 \leq 55-56\end{array}$ \\
\hline
\end{tabular}

Detailes on the volcanoes of Kamchatka, Kurile and Indonesia will be described in another paper. 
Table 10. Number of analyses, range and peaks of frequency distribution based on $\mathrm{SiO}_{2}$, $100 \mathrm{MgO} / \mathrm{Total} \mathrm{FeO}+\mathrm{MgO}+\mathrm{Alk}$ and $100 \mathrm{Alk} / \mathrm{Total} \mathrm{FeO}+\mathrm{MgO}+\mathrm{Alk}$, and alkali-lime index of each group (refer to Table 2)

\begin{tabular}{|c|c|c|c|c|c|c|c|}
\hline \multirow{2}{*}{$\begin{array}{c}\text { Group } \\
\text { No. refer } \\
\text { to Table } 2\end{array}$} & \multirow{2}{*}{$\begin{array}{l}\text { No. of } \\
\text { Anal.* }\end{array}$} & \multirow{2}{*}{$\begin{array}{l}\text { Distri- } \\
\text { bution }\end{array}$} & \multirow{2}{*}{$\mathrm{SiO}_{2}$} & $100 \mathrm{MgO}$ & 100 Alk & \multirow{2}{*}{\multicolumn{2}{|c|}{$\begin{array}{c}\text { Alkali-lime } \\
\text { index } \\
\mathrm{SiO}_{2} \mathrm{CaO}=\mathrm{Alk}\end{array}$}} \\
\hline & & & & $\mathrm{FeO}+\mathrm{MgO}+\mathrm{Alk}$ & $\mathrm{FeO}+\mathrm{MgO}+\mathrm{Alk} \mid$ & & \\
\hline \multirow[t]{3}{*}{1} & \multirow{3}{*}{52} & Range & $50-72$ & $27-8$ & $9-61$ & \multirow{3}{*}{64.4} & \multirow{3}{*}{5.2} \\
\hline & & Peak & $52-53$ & $25-23$ & $10-12$ & & \\
\hline & & & $59-62$ & $20-17$ & $30-34$ & & \\
\hline \multirow[t]{3}{*}{2} & \multirow{3}{*}{80} & Range & $46-73$ & $34-0$ & $10-84$ & \multirow{3}{*}{63.6} & \multirow{3}{*}{5.1} \\
\hline & & Peak & $59-61$ & $24-23$ & $16-18$ & & \\
\hline & & & $51-52$ & $19-17$ & $34-36$ & & \\
\hline \multirow{2}{*}{$\begin{array}{l}\text { Total }(1+2) \\
=\operatorname{active}(\mathrm{a})\end{array}$} & \multirow{2}{*}{132} & Range & $46-73$ & $34-0$ & 9-84 & \multirow[b]{2}{*}{64.0} & \multirow[b]{2}{*}{5.1} \\
\hline & & Peak & $\begin{array}{l}59-61 \\
51-54\end{array}$ & $\begin{array}{l}24-32 \\
19-17\end{array}$ & $\begin{array}{l}10-12 \\
26-28 \\
32-36\end{array}$ & & \\
\hline \multirow{2}{*}{$\begin{aligned} & 3 \\
= & \operatorname{active}(\mathrm{b})\end{aligned}$} & \multirow{2}{*}{196} & Range & $46-77$ & $36-0$ & (6) -92 & \multirow[b]{2}{*}{64.1} & \multirow[b]{2}{*}{5.1} \\
\hline & & Peak & $\begin{array}{l}56-58(61) \\
49-53 \\
60-61\end{array}$ & $\begin{array}{l}28-27 \\
20-19\end{array}$ & $\begin{array}{l}22-24 \\
28-32 \\
10-12\end{array}$ & & \\
\hline \multirow{3}{*}{$\begin{array}{l}\text { Total } \\
(1+2+3)\end{array}$} & \multirow{3}{*}{328} & Range & $46-77$ & $36-0$ & (6) -92 & \multirow{3}{*}{64.1} & \multirow{3}{*}{5.1} \\
\hline & & Peak & $57-61$ & $26-23$ & $22-36$ & & \\
\hline & & & $51-54$ & $21-18$ & $4-16$ & & \\
\hline \multirow{3}{*}{$\begin{array}{l}4 \\
\text { non- } \\
\text { active }\end{array}$} & \multirow{3}{*}{291} & Range & $47-77$ & $40-0$ & $8-84$ & \multirow{3}{*}{63.4} & \multirow{3}{*}{5.2} \\
\hline & & Peak & $50-53$ & $25-12$ & $16-42$ & & \\
\hline & & & $57-65$ & $27-26$ & $10-18$ & & \\
\hline \multirow{3}{*}{$\begin{array}{c}\text { Total } \\
(1+2+3+4)\end{array}$} & \multirow{3}{*}{619} & Range & $46-77$ & $40-0$ & (6) -92 & \multirow{3}{*}{63.8} & \multirow{3}{*}{5.2} \\
\hline & & Peak & $57-61$ & $21-18$ & $(22-) 28-30$ & & \\
\hline & & & $51-53$ & $26-25$ & $16-18$ & & \\
\hline
\end{tabular}

* Two or more data of the same lava flow or the same bomb-pumice bed are taken as average. 


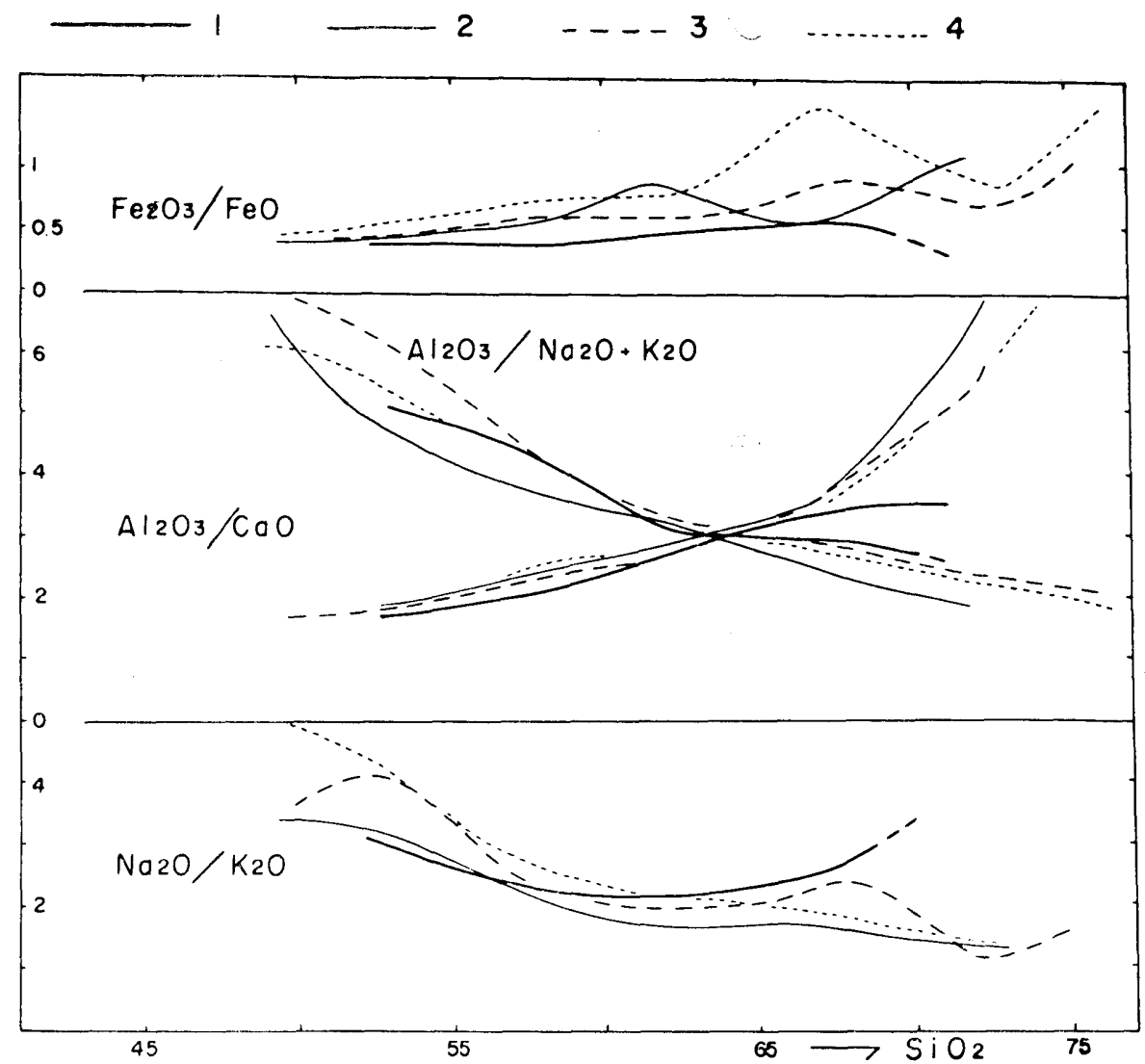

Fig. 5-B. Nos. refer to Table 2.

to be between $59-61 \%$, and the second peak between $51-52 \%$ for those of prehistoric times.

These facts appear to be taken into account together with a similar tendency found in the active volcanoes in Kamchatka, Kurile and Indonesia (see Table 9 \& Fig. 6).

\section{Comparison of active volcanoes (a) and (b), and non-active volcanoes with each other}

Number of analytical data, variation range in chemical composition ( $\mathrm{SiO}_{2}, 100$ $\mathrm{MgO}$ /Total $\mathrm{FeO}+\mathrm{MgO}+\mathrm{Alk}, 100 \mathrm{Alk} /$ Total $\mathrm{FeO}+\mathrm{MgO}+\mathrm{Alk}$ ), alkali-lime index and frequency distribution are given in Table 10, and Figs. 3, 4 and 5. From this Table and these Figs. some points to be noticed are summarised below.

\section{a. Alkali-lime index}

Alkali-lime index of active volcanoes (sum of (a) and (b) of Table 2) is higher than that of non-active volcanoes (4 of Table 2 ). The index of the lavas of historic times is higher than that of lavas of prehistoric times. 


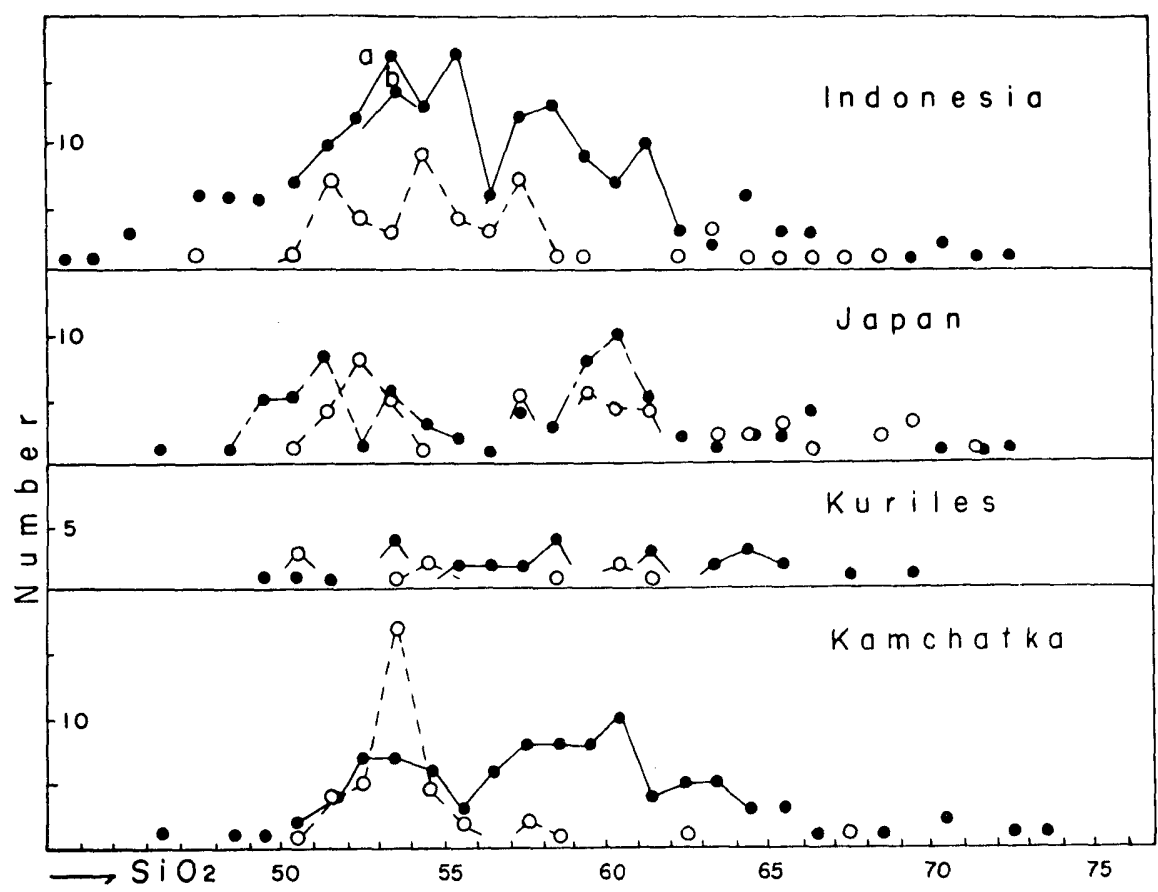

Fig. 6. Frequency diagrams.

Open circles: Lavas of historic times.

Solid circles: Lavas of prehistoric times of all active volcanoes with or without analyses of the lavas of historic times in Indonesia, Kurile and Kamchatka; and the lavas of prehistoric times of Japanese volcanoes which have flowed out lava in historic times.

Here I notice that the alkali-lime index has increased slightly from non-active volcanoes to active ones, and has reached the highest point as opfosed to the lavas of historic times.

\section{b. Successive increase or decrease of some contents}

The values of Total $\mathrm{FeO}$ and $\mathrm{MnO}$ increase successively from the non-active volcanoes to the active ones, and moreover from prebistoric times to historic times. On the other hand the values of $\mathrm{H}_{2} \mathrm{O}+, \mathrm{H}_{2} \mathrm{O}-$ and $\mathrm{Fe}_{2} \mathrm{O}_{3} / \mathrm{FeO}$ decrease successively (Fig. 5).

These facts appear to be significant concerning to sampling of analysed specimens and/or the volcanologic consideration of magma.

\section{c. Frequency distribution}

Frequency distribution of lavas based on the $\mathrm{SiO}_{2}$ percentage seems to be similar to the frequency distribution based on the ratio $\mathrm{Alk} / \mathrm{Total} \mathrm{FeO}+\mathrm{MgO}+\mathrm{Alk}$, though it is rather different from the frequency distribution based on the ratio $\mathrm{MgO} /$ Total $\mathrm{FeO}+\mathrm{MgO}+\mathrm{Alk}$. 


\section{d. Frequency distribution based on the silica percentage}

(1) The curve of frequency distribution of lavas based on the silica percentage for non-active volcanoes (4 in Table 2), shows a rather sharp peak between 50-53, and broadpeak between 57-63, though the curve for active volcanoes (sum of 1,2 and 3 of Table 2) shows the highest peak between 57 and 61 , and the second peak between 51 and 54.

(2) The curve of the lavas of historic times (1 in Table 2) shows the highest peak between 52 and 53 and the second peak between 59 and 62 as previously mentioned.

(3) The curve of the active volcanoes which have never flowed out lavas in historic times ( 3 in Table 2) shows a stout peak between 56 and 59.

(4) It is to be noticed that each of these assymetric frequency curves appear to be a combination of several symmetrical frequency curves, the peaks of which are between 50 and 53,56 and 58,59 and 62, 63 and 65, etc. For convenience the frequency curve having a peak between 50 and 53 is called "curve I" and that having a peak between 56 and 58 is called "curve II", similarly curves III and IV corresponding the peaks of $\mathrm{SiO}_{2} 59-62$ and 63-65 respectively.

In the non-active volcanoes, each curve (I-IV) is almost equal at its height of peak, while in the active volcanoes, the curves I, II, III predominate over curve IV.

The curves III and I predominate in the active volcanoes which have ejected lavas in historic times, though curve II predominates in the other active volcanoes which have never flowed out lavas in historic times. In recent lavas the curves I and III predominate (refer to Table 9).

\section{Suggestion concerning the forecast of volcanism}

The frequency distribution of analysed lavas (lava flows and a few bombs or pumices producod by distinct eruptions) seems to represent, to some extent, the tendency of frequency distribution of lavas of Japanese volcanoes as a whole. If it is real, the facts above mentioned may be usable to presume the fluctuation of activity of magma in an unit area as large as the Japanese Islands throughout pleistocene and holocene. Taking into account all the knowledge about volcanism in Japan, it appears to be true that basic lavas and moderately acid lavas should hold more steady activity, compared with the intermediate lavas, for the present.

It schould be significant that the frequency peaks for lavas are well corresponding to the frequency peaks for volcanoes (Fig. 2-B).

On the other hand, the characteristics of the recent (historic) lavas (compared with the prehistoric lavas) refer mainly to the limited distribution of the active volcanoes in Japan (Taneda, p. 219 (pp. 224-229) in this volume). 
I suppose that as soon as the fluctuation trend of frequency distribution of lavas throughout geologic times, as well as recent times, is clarified, it will be possible to forecast what kind of lava may be ejected at an uncertain or certain place in the future. At a certain condition, it will be available for the estimation of the centre of possible eruption besides the eruption type.

\section{Acknowledgement}

I acknowledge the contributions made by many investigators and analysts. I am indebted to Mr. H. Kurasawa of the Geological Survey of Japan who analysed the lava specimens of Haruna and Aso for me, Prof. H. Kuno of Tokyo University who gave kind criticism, and Prof. T. Tomita who gave facilities for this investigation.

This work has been made on the Grant in Aid for Scientific Researches from the Ministry of Education, Japan.

\section{References}

List of the original papers including analytical results is omitted reluctantly due to the lack of space. (See Table 3).

Taneda, S. (1952): New average chemical compositions of Japanese effusive rocks. Jour. Geol. Soc. Japan. 53, 517-521.

Taneda, S. (1961): Petrochemical study of the volcanic rocks of Indonesia. Sci. Rep. Fac. Sci. Kyushu Univ., (Geol.), 5, 181-195.

Taneda, S. (1962): Petrochemical studies on the active volcanoes in Japan. This Volume, pp. 219. 